J. Comp. Int. Sci. (2016) 7(1):21-25

http://epacis.net/jcis/PDF_JC/S/JC/S-0104.pdf

jcis@epacis.net

(C2016 PACIS (http://epacis.net)

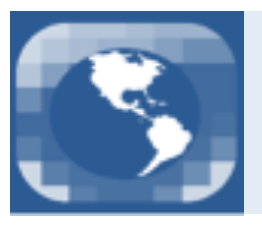

\title{
Probing cosmology in the outskirts of galaxy clusters
}

\author{
A. P. A. Andrade ${ }^{a 1}$, A. L. B. Ribeiro ${ }^{a}$ and P. A. A. Lopes $^{b}$ \\ ${ }^{a}$ Universidade Estadual de Santa Cruz, Ilheus, BA, Brazil \\ ${ }^{b}$ Universidade Federal do Rio de Janeiro, Rio de Janeiro, RJ, Brazil \\ Received on January 31, 2015 / accepted on March 30, 2016
}

\begin{abstract}
We study a sample of 183 galaxy clusters over a scale of $\leq 6 \mathrm{Mpc}$ to investigate the structure of peculiar velocity field around these systems in order to explore the outskirts of galaxy clusters as cosmological probe for dark energy candidates. We find the velocity profiles around the virial radii of the clusters and compare observational data and theory for a $\Lambda$ CDM model and a dynamical dark energy model, such as ChevallierPolarski-Linder parametrization. We find differences higher than 12 percent between $\Lambda$ CDM and CPL models for $R / R_{V} \geq 5.0 \mathrm{Mpc}$. These differences exceeds the $2 \sigma$ margin around the theoretical profile given by the infall collapse model, suggesting that the velocity field in the outskirts of galaxy clusters can be usefull to investigate cosmological models only in observational ranges $\geq 5.0 \mathrm{Mpc}$.
\end{abstract}

Keywords: large scale structures; observational cosmology; model comparison; computational astronomy.

\section{Introduction}

Physical cosmology is the study of the whole universe, concerning about origin, evolution and fate of large scale structure in cosmos. The current big challenge in cosmology is to draw a distinction among different cosmological models of the expanding Universe. The main problem is that cosmological modelling involves a large number of parameters, most of then quite difficult to estimate from observational data. However, in the past decade, the cosmological research has been largely improved by quality and quantity of observational data. Now, a great effort is being done in order to develop appropriate tools to extract information from data and make comparisons against theory.

In this paper, we probe cosmology in the surroundings of galaxy clusters. These systems are the largest gravitationally bound structures in the Universe and constitute an important source of information in modern cosmology. The velocity and spatial distributions of galaxies in clusters are powerful tools to understand the formation and the evolution of cosmic structures (e.g. [1]), as well as they provide constraints on the cosmological parameters, in particular the dark energy equation of state parameter $\omega$ (e.g. [2] \& [3])

Here, we investigate the peculiar velocities in the neighborhoods of a well selected sample of galaxy clusters. We explore these regions to assess the extent to which cosmological parameters can be constrained using the internal dynamics of galaxy clusters and the theoretical expectations from the spherical collapse model for virialized halos. Our aim is to provide pointers to establish that $\omega \neq-1$ or that it changes with time, ruling out vacuum energy as a dark energy candidate.

\footnotetext{
${ }^{1}$ E-mail Corresponding Author: apaula@uesc.br
} 


\section{Data}

This work is based on the supplemental version of the Northern Sky Optical Cluster Survey - NoSOCS [4] \& [5]. This supplemental version of NoSOCS goes deeper $(z \sim 0.5)$, but covers a smaller region $(\sim 2,700$ square degrees) than the main NoSOCS catalog [6] \& [7]. NoSOCS was created from the digitized version of the Second Palomar Observatory Sky Survey - DPOSS/POSS-II [8]. The photometric calibration and object classification for DPOSS are described in Gal et al. [9] and Odewahn et al. [13], respectively. In the first paper of this series [14] a subsample of 7,414 systems from the NoSOCS supplemental was extracted from the Sloan Digital Sky Survey (SDSS), data release 5 (DR5).

This sample was reduced to 179 low redshift clusters $(z \leq 0.100)$ with enough spectra in SDSS (at least three galaxies within $0.50 \mathrm{~h}^{-1} \mathrm{Mpc}$ ) for spectroscopic redshift determination using the gap-technique [11] $\&$ [12]. After the interloper removal the final sample comprises 127 clusters (out of 179) with at least 10 member galaxies.

This sample was complemented with 56 more massive systems from the Cluster Infall Regions in SDSS (CIRS) sample [15]. CIRS is a collection of $z \leq 0.100$ X-ray selected clusters overlapping the SDSS DR4 footprint. In this redshift range, NoSOCS comprises only relatively poor systems. The same cluster parameters listed above were determined for these 56 CIRS clusters. Hence, the combined NoSOCS plus CIRS sample comprises 183 clusters.

The redshift limit of the sample $(z=0.100)$ is due to incompleteness in the SDSS spectroscopic survey for higher redshifts, where galaxies fainter than $M^{*}+1$ are missed, biasing the dynamical analysis (see discussion in section 4.3 of Lopes et al. [14]). We considered the $M^{*}=-20.94$ value from Popesso et al. [16], converted to our cosmology. Our galaxy sample, consisting of 40,323 members from these 183 clusters at $z \leq 0.100$ up to $\sim 6.0 \mathrm{Mpc}$, is an extension of the original NoSOCS data, previously restricted to $\sim 2.5$ $\operatorname{Mpc}[4]$.

\section{Methods}

Conceptual difficulties about the cosmological constant have led to many models where dark energy varies with time. Most of them make use of a homogeneous, time dependent minimally coupled scalar field, $\phi$, whose dynamics is determined by a specific potential, $V(\phi)$, inducing the appropriate equation of state $\omega(z)=p(\phi) / \rho(\phi)$. Such models predict specific forms of the expansion history, E(z), which can be tested against observational data. This has been the basic methodology to identify the best dark energy models. In this work, we consider the commonly used CPL model [17] \& [18], in which $\omega(z)$ is parameterized as

$$
\omega(z)=\omega_{0}+\omega_{a} \frac{z}{(1+z)}
$$

where $\omega_{0}$ and $\omega_{a}$ are constants. Under the assumption of a flat Universe, the corresponding $E(z)$ is expressed as

$$
E(z) \approx \sqrt{\Omega_{m}(1+z)^{3}+\left(1-\Omega_{m}\right)(1+z)^{3\left(1+\omega_{0}+\omega_{a}\right)} \exp \left(-\frac{3 \omega_{a} z}{1+z}\right)} .
$$

Recent studies [19] based on joint data analysis indicate that the best-fit parameters are: $\Omega_{m}=0.265$, $\omega_{0}=-0.847, \omega_{a}=-0.691$, and $h=0.716$. Note that the best-fit parameters of the $\Lambda$ CDM model are $\Omega_{m}=0.275, \omega_{0}=-1$, and $\omega_{a}=0$ (e.g. [19]) In this context, a significant progress is expected from establishing that $\omega_{0} \neq-1$, ruling out vacuum energy as a dark energy candidate.

In this work, we face this problem by using statistical tools over galaxy clusters data. Our method consists of two steps: (i) an initial investigation of the peculiar velocity profiles around the NoSOCS clusters in each model, aiming to find significant differences between them; and (ii) model the differences as a function of the radius using a modified version of the Barkana code to study the infall around virialized halos [20]. Since the collapsed part of the halo has both $R_{V}$ and $V_{C}$ dependent on cosmology in the theory of halo collapse, we have to compute these quantities in the two models considered here. In fact, 


$$
R_{V} \simeq R_{200}=\frac{\sqrt{3} \sigma_{v}}{10 H(z)} \text { and } V_{C} \simeq \sqrt{\frac{G M}{r_{200}}}
$$

where $\sigma_{v}$ is the line-of-sight velocity dispersion, $H(z)=H_{0} E(z)$, and $\mathrm{G}$ is the gravitational constant. At the same time [21]:

$$
M=3 \frac{\sigma_{v}^{2} R_{200}}{G} .
$$

The dependence of these quantities on cosmology makes it more difficult to compare the model with theory. In our working method, we are searching for significant differences in the radial profile of the ratio of peculiar velocities around clusters $V_{p e c}$ (a kinematical quantity given by $v_{i}-v_{c l} ; v_{i}$ is the velocity of each galaxy with respect to the cluster velocity $v_{c l}$ ) to the circular velocity $V_{C}$ at the virial radius (a dynamical quantity). To do this, we find $\Delta V_{p e c} / V_{C}$ for both theory and data. In this preliminary analysis we assume significant differences as those higher than $2 \sigma$. Our results are discussed in the next section.

\section{Discussion}

In Figure 1 we present our results for $\Delta V_{p e c} / V_{C}$ around the NoSOCS clusters. Points indicate the differences for the observational stacked sample due to cosmological dependence. Errors bars are the counting errors weighted by the number of galaxies in each bin. The solid black line refers to the absolute difference between the $\Lambda \mathrm{CDM}$ and CPL models. Dashed lines mark the $2 \sigma$ contours around the theoretical curve. The red vertical dotted line indicates the radius at which the two models start to present significant differences for the NoSOCS sample.

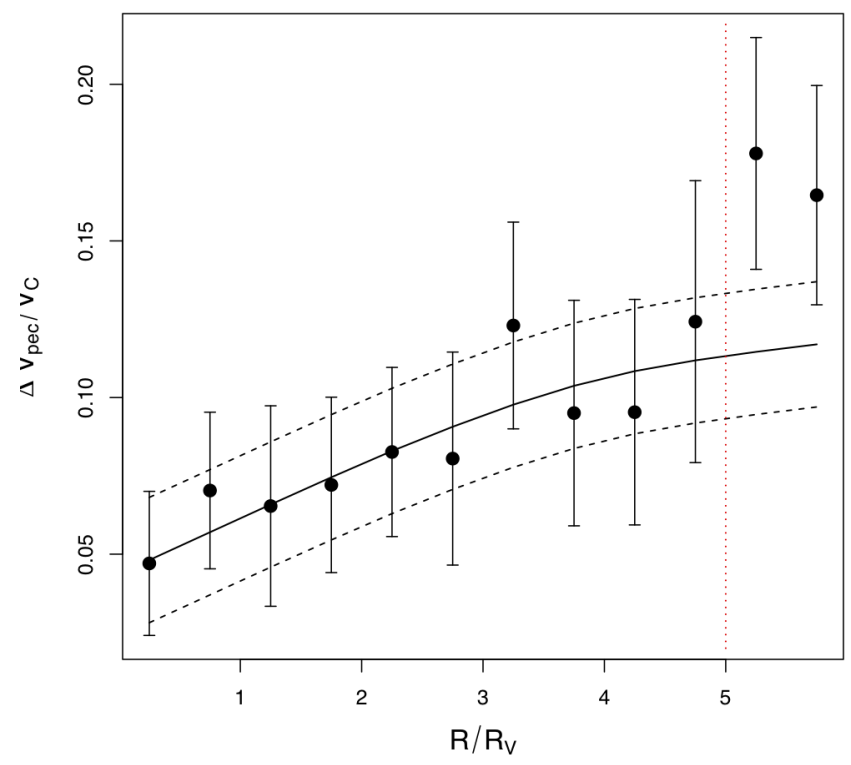

Figure 1: Differences in the velocity profiles around the NoSOCS clusters. $V_{p e c}$ is the peculiar velocity (negative values correspond to infall) at the radius $R$. $V_{C}$ is the circular velocity at the virial radius $R_{V}$. The solid black line refers to the absolute difference between the $\Lambda$ CDM and the CPL models. Dashed lines mark the $2 \sigma$ contours around the theoretical curve. The red vertical dotted line indicates the radius at which the two models start to present significant differences.

We should note that $\Delta V_{p e c} / V_{C}$ is an increasing function with radius. Interestingly, for $R / R_{V} \leq 5.0 \mathrm{Mpc}$, differences between $\Lambda \mathrm{CDM}$ and CPL models achieve levels of around 5-12 percent, but still remain within the $2 \sigma$ margin around the theoretical profile. Only for greater distances we find differences in excess of this 
threshold, which means that the comparison requires differences higher than 12 percent to allow its use in cosmology.

\section{Conclusion}

We investigate the peculiar velocities in the neighborhoods of a well selected sample of galaxy clusters. We explore these regions up to 6.0 Mpc around the NoSOCS galaxy clusters to assess the extent to which cosmological parameters can be constrained using the internal dynamics of galaxy clusters and the theoretical expectations from the spherical collapse model of virialized halos. Our aim is to establish that $\omega_{0} \neq-1$, ruling out vacuum energy as a dark energy candidate. Our preliminary results indicate that the $\Delta V_{\text {pec }} / V_{C}$ profile is an increasing function with radius, with significant differences between $\Lambda$ CDM and CPL models for $R / R_{V} \leq 5.0 \mathrm{Mpc}$. This suggests that the velocity field in the outskirts of galaxy clusters can be used to exploratory analysis of cosmological models. However, the procedure requires updating to break the degeneracy due to cosmological dependence on the computation of $R_{V}$ and $V_{C}$. This can be solved through a kinematical attainment of these quantities. For instance, using the projected virial radius (e.g. [22]) this can be achieved. This update, as well as the exploration of greater distances around the cluster centers, will be tackled in future research.

\section{References}

[1] Menci, N; Fusco-Femiano, R; 1996, ApJ, 472, 46

[2] Haiman, Z; Mohr, JJ; Holder, GP; 2001, The Astrophysical Journal, Volume 553, Issue 2, pp. 545-561

[3] Cunha, C; Huteres, D; Frieman, JA; 2009, Physical Review D, vol. 80, Issue 6, id. 063532

[4] Lopes, PAA; 2003, Ph.D. Thesis, Observatório Nacional (Brazil)

[5] Lopes, PAA; de Carvalho RR; Gal RR; Djorgovski SG; Odewahn SC; Mahabal AA; Brunner RJ; 2004, AJ, 128, 1017

[6] Gal, RR; de Carvalho, RR; Lopes, PAA; Djorgovski SG; Brunner R.J; Mahabal AA; Odewahn SC; 2003, AJ, 125, 20642003

[7] Gal, RR; Lopes, PAA; de Carvalho, RR; Kohl-Moreira, JL; Capelato, HV; Djorgovski, SG; 2009, AJ, 137,2981

[8] Djorgovski SG; de Carvalho RR; Gal RR; Odewahn SC; Mahabal AA; Brunner RJ; Lopes PAA; KohlMoreira JL; 2003, Bulletin of the Astronomical Society of Brazil, 23, 197

[9] Gal RR; de Carvalho RR; Odewahn SC; Djorgovski SG; Mahabal AA; Brunner RJ; Lopes PAA; 2004, AJ, 128, 3082

[10] Lopes, PAA; de Carvalho, RR; Kohl-Moreira, JL; Jones, C; 2009, MNRAS, 392, 135

[11] Katgert et al; 1996, A\&A, 310, 8

[12] Lopes, PAA; 2007, MNRAS, 380, 1680

[13] Odewahn SC; de Carvalho RR; Gal RR; Djorgovski SG; Brunner RJ; Mahabal AA; Lopes PAA; KohlMoreira JL; Stalder B; 2004, AJ, 128, 3092

[14] Lopes, PAA; de Carvalho, RR; Kohl-Moreira, JL; Jones, C; 2009, MNRAS, 392, 135

[15] Rines, K; Diaferio, A; 2006, AJ, 132, 1275 
[16] Popesso, P; Biviano, A; Böhringer, H; Romaniello M; 2006, A\&A, 445, 29

[17] Chevalier, M; Polarski, D; 2001, Int.J.Mod.Phys.D,10, 213

[18] Linder, VE; 2003, Phys. Rev. Lett., 90, 091301

[19] Li, M; Li, X; Zhang X; 2010, Science China, 53, 9, 1631

[20] Barkana, R; 2004, Mon.Not.Roy.Astron.Soc. 347, 57

[21] Carlberg, RG; Galaxies in the Young Universe, Lecture Notes in Physics, 1996, 463, 2061996

[22] Girardi, M; Biviano, A; Giuricin, G; Mardirossian, F; Mezzetti, M; 1995, ApJ, 438, 527 